\title{
Enhancement of Radiosensitivity by DNA Hypomethylating Drugs through Apoptosis and Autophagy in Human Sarcoma Cells
}

\author{
Moon-Taek Park ${ }^{1}$, Sung-Dae Kim ${ }^{1}$, Yu Kyeong Han ${ }^{2}$, Jin Won Hyun ${ }^{3}$, Hae-June Lee ${ }^{4}$ and Joo Mi Yi,* \\ ${ }^{1}$ Research Center, Dongnam Institute of Radiological \& Medical Sciences (DIRAMS), Busan 46033, \\ ${ }^{2}$ Department of Microbiology and Immunology, College of Medicine, Inje University, Busan 47392, \\ ${ }^{3}$ Jeju National University School of Medicine and Jeju Research Center for Natural Medicine, Jeju 63243, \\ ${ }^{4}$ Division of Radiation Biomedical Research, Korea Institute of Radiological \& Medical Sciences, Seoul 01812, Republic of Korea
}

\begin{abstract}
The targeting of DNA methylation in cancer using DNA hypomethylating drugs has been well known to sensitize cancer cells to chemotherapy and immunotherapy by affecting multiple pathways. Herein, we investigated the combinational effects of DNA hypomethylating drugs and ionizing radiation (IR) in human sarcoma cell lines both in vitro and in vivo. Clonogenic assays were performed to determine the radiosensitizing properties of two DNA hypomethylating drugs on sarcoma cell lines we tested in this study with multiple doses of IR. We analyzed the effects of 5-aza-dC or SGI-110, as DNA hypomethylating drugs, in combination with IR in vitro on the proliferation, apoptosis, caspase-3/7 activity, migration/invasion, and Western blotting using apoptosis- or autophagy-related factors. To confirm the combined effect of DNA hypomethylating drugs and IR in our in vitro experiment, we generated the sarcoma cells in nude mouse xenograft models. Here, we found that the combination of DNA hypomethylating drugs and IR improved anticancer effects by inhibiting cell proliferation and by promoting synergistic cell death that is associated with both apoptosis and autophagy in vitro and in vivo. Our data demonstrated that the combination effects of DNA hypomethylating drugs with radiation exhibited greater cellular effects than the use of a single agent treatment, thus suggesting that the combination of DNA hypomethylating drugs and radiation may become a new radiotherapy to improve therapeutic efficacy for cancer treatment.
\end{abstract}

Key Words: DNA hypomethylating drugs, 5-aza-dC, SGI-110, lonizing radiation, Apoptosis, Autophagy

\section{INTRODUCTION}

Sarcoma is a heterogeneous group comprised of over 160 different bone and soft tissue neoplasms and categorized more than 60 malignancies in terms of diverse biological and clinical characteristics (Gage et al., 2019). Therefore, the variability of all of the subtypes of sarcomas is not well defined due to the heterogeneity of the disease, with various subtypes in treatment responses, behavior, and biology (Toro et al., 2006; Brennan et al., 2014; Kneisl et al., 2014; Trans-Atlantic Retroperitoneal Sarcoma Working Group, 2018). Although the etiology associated with the pathogenesis of sarcomas is largely unknown, recent decades have expanded on the understanding of the underlying biological and genetic alterations of many sarcoma subtypes and found that the genetics of sarcoma are highly variable. Some types of sarcoma exhibit simple genetic characteristics driven by chromosomal translocations that lead to fusion oncogenes, including Ewing sarcoma and synovial sarcoma. Other types of sarcomas have complex genomic alterations, such as osteosarcoma, which is driven by copy number alterations or gene mutations (Schaefer et al., 2017). Recently, there has been increasing evidence that many types of sarcoma are associated with widespread epigenetic dysregulation (Jain et al., 2010; Schaefer et al., 2017).

The transcriptional gene silencing is regulated by epigenetic mechanisms such as the DNA methylation, the histone modification, and alterations of nucleosome positions at the DNA level. Unlike genetic events, epigenetic events are reversible, which makes epigenetic regulation extremely interesting from the point of view in developing new therapeutic technologies

\section{Open Access https://doi.org/10.4062/biomolther.2021.174}

This is an Open Access article distributed under the terms of the Creative Commons Attribution Non-Commercial License (http://creativecommons.org/licenses/by-nc/4.0/) which permits unrestricted non-commercial use, distribution, and reproduction in any medium, provided the original work is properly cited.
Received Nov 12, 2021 Revised Nov 17, 2021 Accepted Nov 18, 2021 Published Online Dec 10, 2021

\section{${ }^{*}$ Corresponding Author}

E-mail: jmyi76@inje.ac.kr

Tel: +82-51-890-6734, Fax: +82-51-891-6004 
for cancer treatment. In recent decades, DNA hypomethylating drugs (decitabine and 5-aza-2'-deoxycytidine, also known as 5-aza-dC) have been reported to have anticancer activities in patients with leukemia, myelodysplastic syndrome (MDS), and some other solid tumors (Kaminskas et al., 2005; Yoo et al., 2006). Most recently, SGI-110 (Guadecitabine), which is a second-generation hypomethylating prodrug and a dinucleotide that combines 5-aza-dC and deoxyguanosine (Astex Pharmaceuticals, Inc., Cambridge, UK) has been shown to be less susceptible to deamination by cytidine deaminase and more stable in water solutions (Yoo, 2007), thus convincing it an alternative to 5-aza-dC so far. Many reports have indicated that hypomethylating drugs are a critical chemotherapy that plays through epigenetic mechanisms to target cancer instead of inducing general toxicity, as has been seen with other chemotherapies (Sato et al., 2017). In addition, a few studies have revealed that the combination of these epigenetic regulation with traditional chemotherapy or radiotherapy could improve the therapeutic technique (Kristensen et al., 2009; Kelly et al., 2010).

lonizing radiation is one of the conventional approaches for local control, and the effects of preoperative radiotherapy in sarcomas include reducing tumor size before surgery and decreasing the risk of local recurrence (Ta et al., 2009). However, sarcoma patients always present with radio-resistance, which consequently prevents successful treatment. Therefore, there has not been progress in the radiotherapy of human sarcoma in terms of increasing the tumor response to irradiation with targeted sensitizers.

In the present study, we investigated the combined effect of radiation with epigenetic inhibitors (particularly, DNA hypomethylating drugs) in sarcoma cell lines and explored the biological mechanisms of radiosensitization that is mediated by epigenetic regulation in human sarcoma cells.

\section{MATERIALS AND METHODS}

\section{Cell culture, drug treatment, and irradiation}

Human sarcoma cell lines (SK-LMS-1, SK-UT-1, SK-UT1B, and SK-ES-1) were obtained from American Type Culture Collection (Manassas, VA, USA). Cells were grown in MEM (Welgene, Daegu, Korea) containing 10\% fetal bovine serum (HyClone Laboratories Inc, Logan, UT, USA) and 1\% antibiotic-antimycotic (Thermo Fisher, Waltham, MA, USA) and maintained at $37^{\circ} \mathrm{C}$ in an atmosphere of $20 \% \mathrm{O}_{2}$ and $5 \% \mathrm{CO}_{2}$ incubator. Cells were treated with 5 -aza-dC $(0.1,0.5$ or $1 \mu \mathrm{M})$ (Sigma-Aldrich Chemical Co., St. Louis, MO, USA) or SGI-110 (0.5 or $1 \mu \mathrm{M})$ (APExBio, Houston, TX, USA) once daily for 3 days. Cells were incubated with 5 -aza-dC or SGI-110 for 3 days before and $24 \mathrm{~h}$ after irradiation. Cells that were treated with 5-aza-dC or SGI-110 for 3 days without exposure to radiation or those cells that were solely exposed to irradiation were used as controls. Irradiation (gamma-rays) of cells was performed with a ${ }^{137} \mathrm{Cs}$ ray source (Eckert \& Ziegler, Berlin, Germany) at a dose rate of $2.6 \mathrm{~Gy} / \mathrm{min}$.

\section{Clonogenic assays}

Cells were seeded in 6-well plates (5,000 cells/well) and treated with 5-aza-dC or SGI-110. After drug treatment, cells were irradiated at the specific doses. Twelve to fourteen days after seeding, colonies were fixed and stained with $1.25 \%$ crystal violet. The number of colonies containing at least 50 cells was determined.

\section{Cell proliferation assay and cell viability assay}

Cell proliferation was determined by using a CCK- 8 assay (Dojindo Molecular Technologies, Rockville, MD, USA). Cells $\left(2 \times 10^{5}\right.$ cells $/$ well $)$ were seeded in 6 -well plates and incubated at $37^{\circ} \mathrm{C}$. After $48 \mathrm{~h}$, the cells were washed twice with PBS, and CCK- 8 reagent and culture media (1:1 ratio) were added to each well for $1 \mathrm{~h}$. After the reaction, the absorbance range from $450 \mathrm{~nm}$ was measured by using a Spectra Max (Molecular Devices, San Jose, CA, USA).

\section{Apoptosis assay}

The evaluation of cell apoptosis was performed by using flow cytometry. DNA hypomethylating drugs or irradiated cells were assessed by using an Annexin V/FITC Apoptosis Detection kit (BD Biosciences, Franklin Lakes, NJ, USA). The cells were stained with FITC, Annexin V, and 7-amino-actinomycin (7-AAD), after which they were incubated for $15 \mathrm{~min}$ in the dark.

\section{Western blot analysis}

Cells were lysed with cell lysis buffer. Equal amounts of total proteins were loaded onto $4-12 \%$ SDS-PAGE gels and transferred to PVDF membranes (GE Health care Life Sciences, Marlborough, MA, USA). The membranes were blocked with $5 \%$ skim milk in TBST buffer and incubated with primary antibodies - anti-DNMT1 (Abcam, Cambridge, UK), anticleaved caspase 3, anti-E-cadherin, anti-cleaved caspase 9, anti-cleaved PARP1, anti-ATG3, anti-ATG5, anti-ATG7, antiATG12, anti-ATG16L1 (Cell Signaling Technology, Denvers, MA, USA), anti-LC3A (Novus Biologicals, Littleton, CO, USA), anti-Beclin (BD Biosciences), and anti- $\beta$-actin (Protein Tech group, Rosemont, IL, USA) overnight at $4^{\circ} \mathrm{C}$. The membranes were subsequently incubated with specific horseradish peroxidase-conjugated secondary antibodies. Protein bands were visualized by using a Al600 system (GE Healthcare, Marlborough, MA, USA).

\section{Caspase 3/7 activity assay}

Measurements of caspase 3/7 activities in cells were performed using the commercially available Caspase-Glo 3/7 Assay Kit (Promega, Madison, WI, USA) according to the manufacturer's instructions.

\section{Migration and invasion assay}

Cell migration was determined by using transwell plates ( 8 $\mu \mathrm{m}$ pore size, Corning Costar, Merck, Darmstadt, Germany), and invasion assays were performed by using Matrigel-coated invasion chambers ( $8 \mu \mathrm{m}$ pore size, Corning Costar, Merck). The upper chamber contained osteosarcoma cells in serumfree medium, and the lower chamber contained MEM supplemented with $10 \%$ FBS. Both migration and invasion assays were performed following the manufacturer's instructions. Photographs were taken using a Qlcam image camera system mounted on a Nikon ECLIPSE 80i microscope (Nikon, Minato, Tokyo, Japan).

\section{In vivo subcutaneous xenograft models}

Six-week-old male athymic nude mice obtained from OrientBio (Seongnam, Korea) were maintained with freely pro- 
vided sterile food and water under specific pathogen-free conditions. After a week-long adaptation period to the new environment, the mice were randomized into nine different groups before tumor implementation ( $n=3$ mice per group). All of the animal experimental procedures were reviewed and approved by the Institutional Animal Care and Use Committee of Dongnam Institute of Radiological and Medical Sciences (DIRAMS) (DI-2019-009). To establish the tumor xenograft models, SK-UT-1 or SK-LMS-1 cancer cells (which were treated as indicated) were subcutaneously injected $\left(2 \times 10^{6}\right.$ cells in $200 \mu \mathrm{L}$ PBS) into the flank of each mouse. Tumor growths were measured 2 times a week. Tumor volume $\left(\mathrm{mm}^{3}\right)$ was calculated via the " $\left(\mathrm{W}^{2} \times \mathrm{L}\right) / 2$ " formula (Faustino-Rocha et al., 2013). Twoway ANOVA was used to compare the tumor volume in mice treated with the combination therapies versus those treated with either agent alone. At the termination of the experiment, tumors were retrieved from the euthanized mice to measure the tumor weights. Volumes and weights of the tumors are expressed as the mean \pm SEM.

\section{Statistical analysis}

The results in this study are presented as the mean \pm standard deviation. The significance of data was estimated using either two tailed paired or unpaired student's t test and ANOVA. $p$ values of $<0.05$ were considered to be statistically significant. All statistical analyses were performed using GraphPad Prism 6.0 (GraphPad Prism software Inc., San Diego, CA, USA).

\section{RESULTS}

\section{Low-dose DNA hypomethylating drugs can change the level of DNMT1 in human sarcoma cells}

First, we determined whether sarcoma cell lines affected the protein expression of well-known regulators of DNA methylation, such as DNA-methyltransferase 1 (DNMT1), via the classical DNA hypomethylating drug (5-aza-dC) or secondgeneration DNA hypomethylating prodrug (SGI-110). By using several doses $(0.1,0.5$, and $1 \mu \mathrm{M})$ of these drugs, Western blot analyses showed that the protein levels of DNMT1 were significantly decreased in sarcoma cells after treatment with these two DNA hypomethylating drugs. Interestingly, we found that a low dose $(0.1 \mu \mathrm{M})$ of these drugs was sufficient to induce enough epigenetic inhibition in sarcoma cell lines; accordingly, a low dose $(0.1 \mu \mathrm{M})$ was chosen for subsequent experiments to examine the combined effects of DNA hypomethylating drugs (5-aza-dC or SGI-110) and IR (Fig. 1).

\section{DNA hypomethylating drugs and sarcoma cell radiosensitivity}

To characterize whether 5-aza-dC or SGI-110 increased the sensitivity of sarcoma cell lines to IR, we performed conventional clonogenic assays to compare the cytotoxic effects of 5-aza-dC or SGI-110, IR at different doses by itself, and combination treatments of each DNA hypomethylating drug and IR in four different sarcoma cell lines (SK-LMS-1, SK-UT-1, SK-UT-1B, and SK-ES-1). Sarcoma cells were pretreated with 5-aza-dC $(0.1 \mu \mathrm{M})$ or SGI-110 $(0.1 \mu \mathrm{M})$ for $72 \mathrm{~h}$ before being exposed to various doses of IR $(0.1,0.5,2,4$, and 6 Gy), including a lower dose, without knowing the cytotoxicity level that was induced by IR, and the cells were plated to assess the colony-forming efficiency. In response to IR, there was a dosedependent reduction in cell survival with or without DNA hypomethylating drugs (5-aza-dC or SGI-110). After pretreatment with 5-aza-dC or SGI-110, radiation-induced cell death was significantly increased, accompanied by the formation of few and small colonies in only SK-LMS-1 and SK-UT-1 [(Fig. 2A) but not in SK-UT-1B and SK-ES-1 (data not shown, Supplementary Fig. 1)] cell lines, compared to those in the IR control groups $(p<0.01)$. For the SK-LMS- 1 cells, there was a $24 \%$ decrease in the number of colonies formed after treatment with 5-aza-dC $(0.1 \mu \mathrm{M})$ alone, a $54 \%$ decrease after treatment with SGI-110 (0.1 $\mu \mathrm{M})$ alone, and 1\% (0.1 Gy), 5\% (0.5 Gy), 20\% (2 Gy), 53\% (4 Gy), and 83\% (6 Gy) decreases after exposure to IR alone; in addition, a $40-94 \%$ or $59-94 \%$ decrease in the number of colonies was elicited after the combination treatment of 5 -aza-dC or SGI-110 with IR, respectively $(p<0.01)$. For the SK-UT-1 cells, there was no decrease in the number of colonies that was formed after treatment with 5-aza-dC alone, as well as a $20 \%$ decrease after treatment with SGI110 alone, a $1-84 \%$ decrease after exposure to IR alone, and a $31-97 \%$ or $47-95 \%$ decrease in the number of colonies that was formed after the combination treatment of 5-aza-dC or SGI-110 with IR, respectively $(p<0.001)$. These observations indicated that the radiosensitization that was caused by 5 -aza$\mathrm{dC}$ or SGI-110 in sarcoma cell lines was statistically significant at radiation doses higher than $4 \mathrm{~Gy}(p<0.01)$ (Fig. 2A).

\section{The combination of DNA hypomethylating drugs and IR inhibits cell proliferation in vitro and in vivo}

To evaluate whether 5-aza-dC or SGI-110 in combination with IR affects cell proliferation, we performed a CCK-8 assays in two sarcoma cell lines (SK-LMS-1 and SK-UT-1) because there was no significant change in the number of colonies that was formed after combination treatment with 5-aza-dC, SGI110 , and IR in the other two cell lines. In addition, low doses ( 0.1 and $0.5 \mathrm{~Gy}$ ) of sole IR exposure to both sarcoma cell lines indicated no significant changes in cell growth compared to

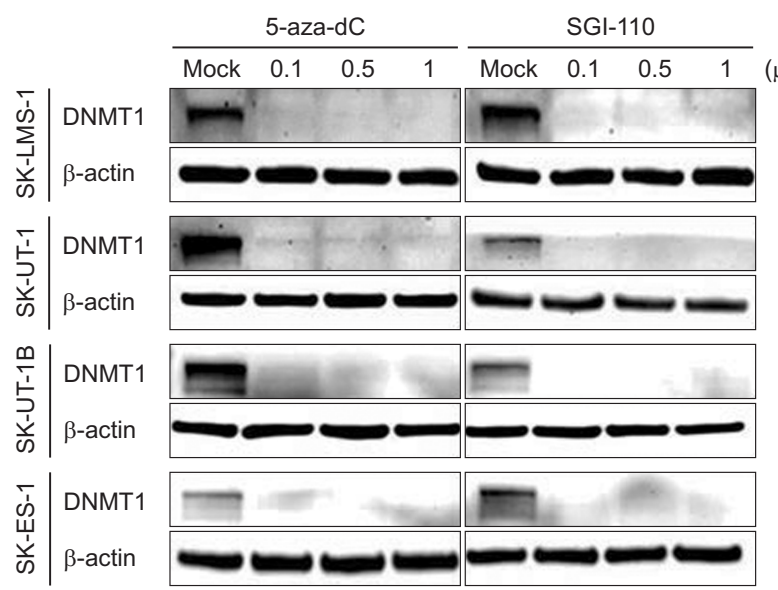

Fig. 1. Low-dose 5-aza-dC and SGI-110 treatments can induce demethylation in human sarcoma cell lines. Western blots show the protein expression levels of DNMT1 $(180 \mathrm{kD})$ in human sarcoma cell lines (SK-LMS-1, SK-UT-1, SK-UT-1B, and SK-ES-1) treated with different doses $(0.1 / 0.5 / 1 \mu \mathrm{M})$ of 5 -aza-dC or SGI-110. The blots were probed by using anti-DNMT1 (upper panel) and anti- $\beta$ actin antibodies. 
A
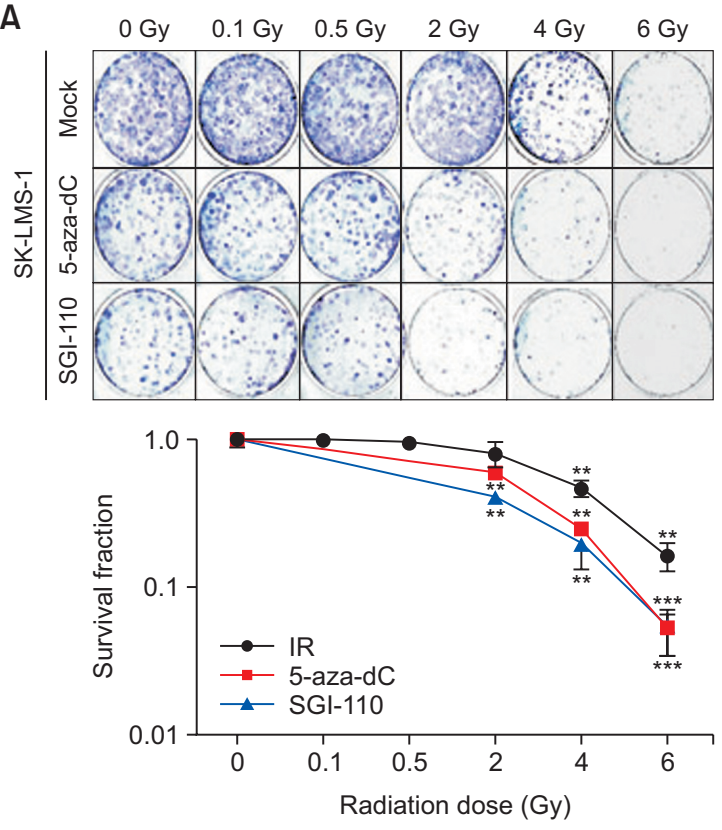

B

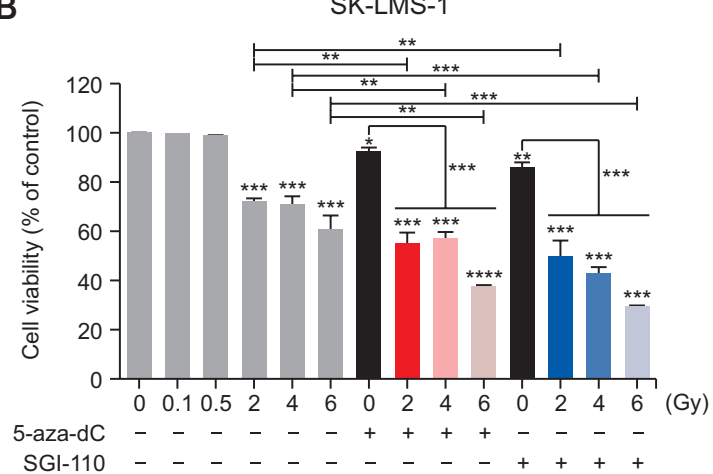

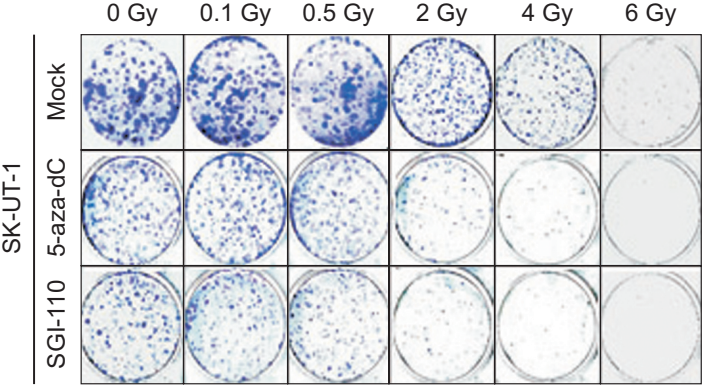

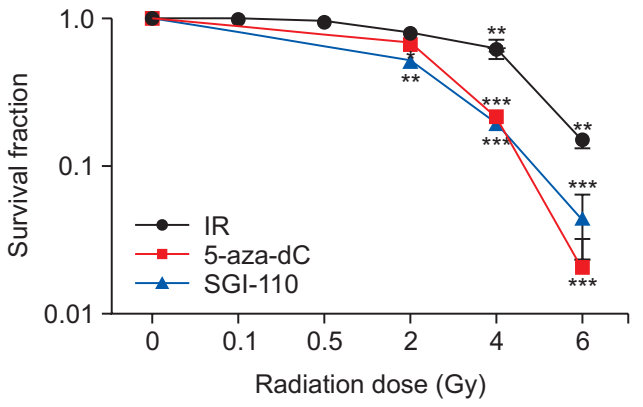

SK-UT-1

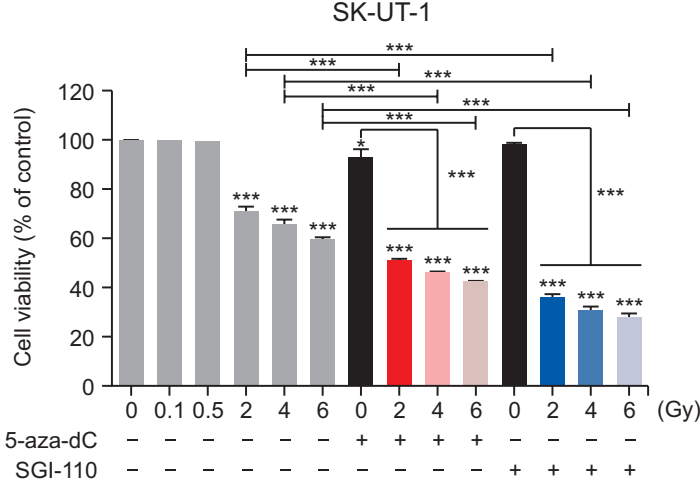

Fig. 2. Combination effects of SK-LMS-1 and SK-UT-1 cells treated with 5-aza-dC or SGI-110, either alone or with IR. (A) Clonogenic death of SK-LMS-1 and SK-UT-1 cells that were treated with 5-aza-dC $(0.1 \mu \mathrm{M})$ or SGI-110 $(0.1 \mu \mathrm{M})$ and/or IR $(2,4$, and 6 Gy). Cells were seeded into 6-well plates $(5,000$ cells/well) and treated with 5 -aza-dC or SGI-110, either alone or in combination with IR, and clonogenic survival was determined. After 2 weeks, cultures were fixed with ethanol and stained with $1.25 \%$ crystal violet. Representative photographs of the single colonies are also shown. (B) CCK-8 assays in SK-LMS-1 (left) and SK-UT-1 (right) cell lines that were treated with 5-aza-dC (0.1 $\mu$ M) or SGI-110 $(0.1 \mu \mathrm{M})$ and/or irradiation $(2,4$, and $6 \mathrm{~Gy})$. Data are expressed as the mean \pm standard deviation of the triplicate experiments. Statistical analysis data are presented as the means $\pm \mathrm{SD}, \mathrm{n}=3 .{ }^{*} p<0.05,{ }^{* *} p<0.01,{ }^{* * *} p<0.001$ compared with control or single treatments.

control cells, thus we excluded the combination treatment of 5 -aza-dC or SGI-110 in subsequent experiments. For the SKLMS-1 cells, there was a 1-39\% decrease in proliferation after treatment with 5-aza-dC, SGI-110, and IR alone, as well as a $43-70 \%$ decrease in proliferation after combination treatment with 5 -aza-dC or SGI-110 and IR $(p<0.001)$. Interestingly, a similar effect was observed in SK-UT-1 cells $(p<0.001)$ (Fig. $2 \mathrm{~B})$. These results indicate that the effects of 5 -aza-dC or SGI110 and IR on growth inhibition are additive.

Based on in vitro data indicating significant growth inhibition of cells treated with the combination of DNA hypomethylating drugs (5-aza-dC or SGI-110) and IR, we investigated whether tumor growth inhibition could be observed in vivo. To investigate the anticancer efficacy of 5-aza-dC or SGI-110 in vivo, xenograft studies were performed in athymic nude mice. Both SK-LMS-1 and SK-UT-1 cells were pretreated with 5-aza-dC
$(0.1 \mu \mathrm{M})$ or SGI-110 $(0.1 \mu \mathrm{M})$ for $72 \mathrm{~h}$ before exposure to IR $(2$ and $4 \mathrm{~Gy}$ ), after which the cells were subcutaneously injected into athymic nude mice. In this experiment, only 2 and 4 Gy IR doses were chosen because there was a similar combination effect between 4 and 6 Gy from in vitro data, thus demonstrating that a relatively lower dose of IR could induce a sufficient combination effect involving radiation and epigenetic inhibitors. Fig. $3 \mathrm{~A}$ shows the significant tumor growth delay in response to the combination treatment with 5-aza-dC or SGI110 and IR ( 2 and $4 \mathrm{~Gy}$ ) compared with tumor growth of single treatment with either 5-aza-dC, SGI-110, or IR. In addition, a significantly different tumor size from both SK-LMS-1 and SKUT-1 cells that were treated with both 5-aza-dC and IR was observed compared to that of tumors that were established from both cells that were treated with either 5-aza-dC or IR alone (Fig. 3B). Strikingly, similar effects were observed after 
SK-LMS-1
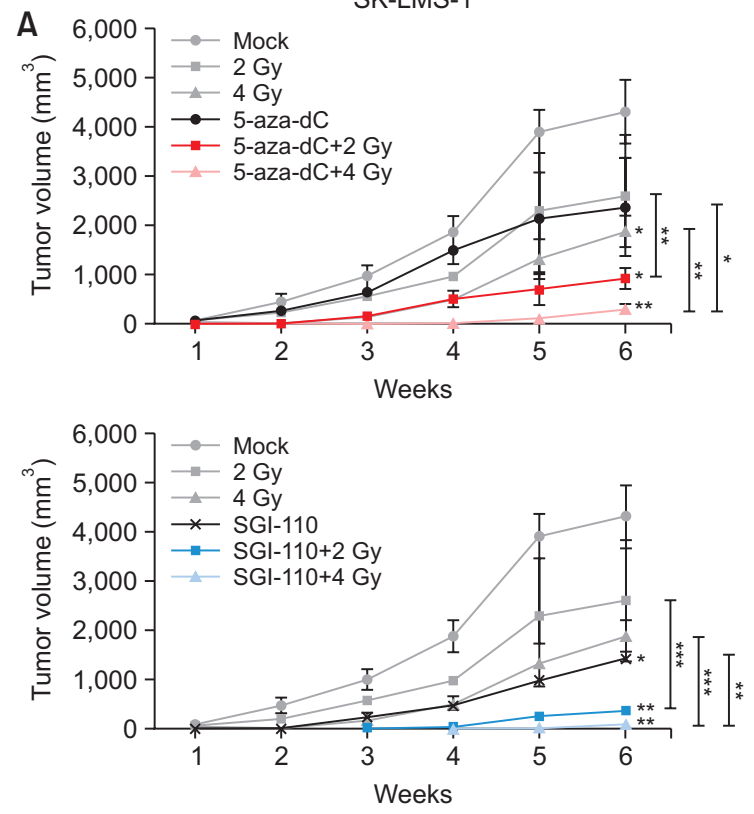

B
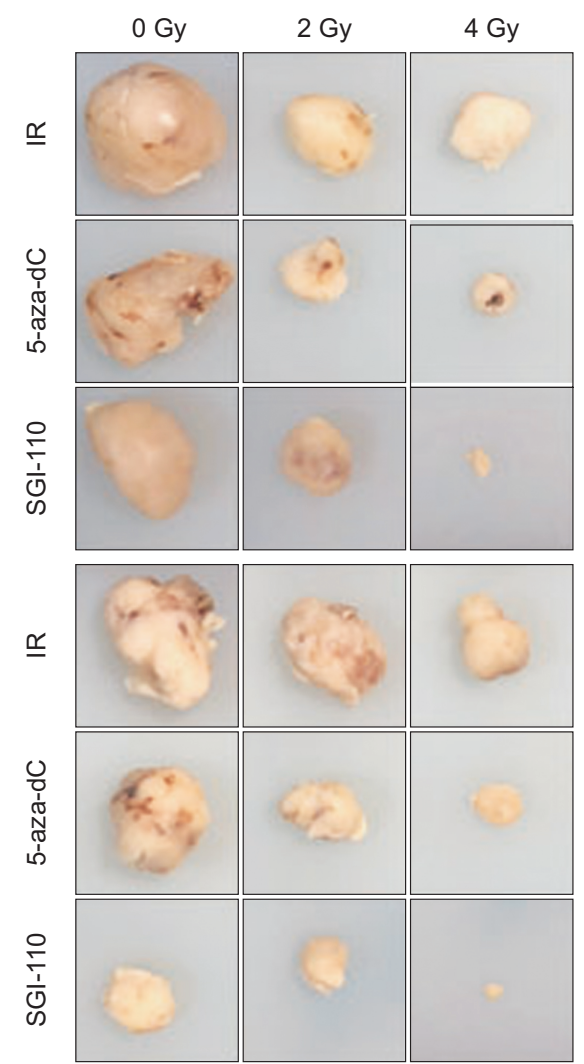

गण
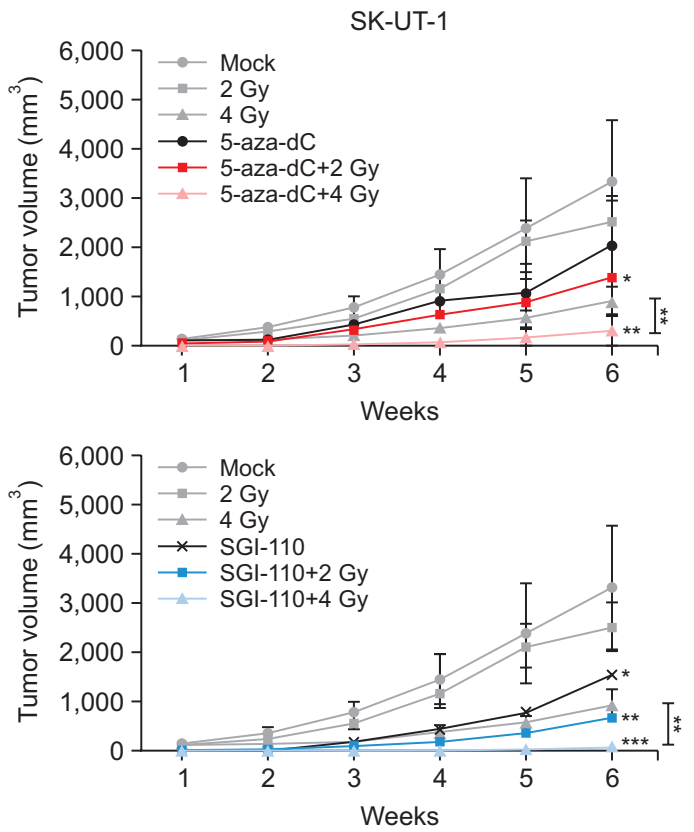

SK-LMS-1

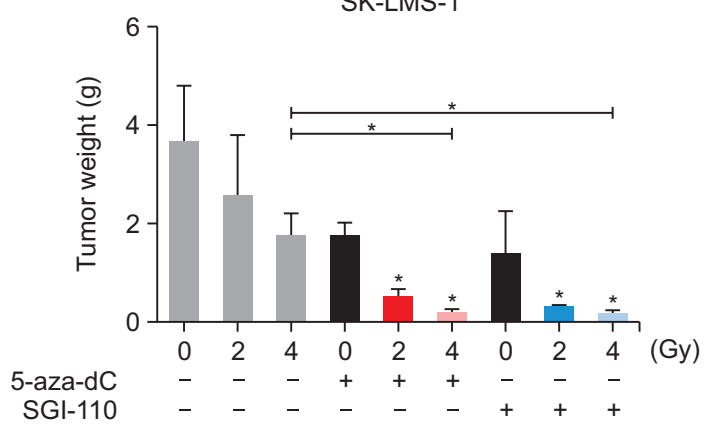

SK-UT-1

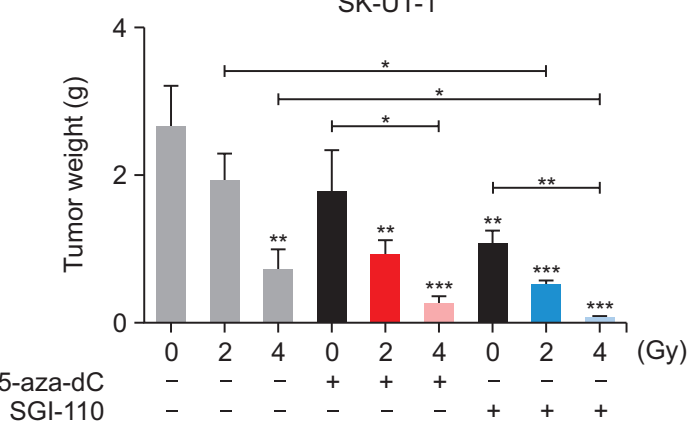

Fig. 3. The anticancer effect of the combination of DNA hypomethylating drugs and IR in the SK-LMS-1 and SK-UT-1 xenograft tumor models. Tumor growth was measured following 5 -aza-dC or SGI-110 treatments either alone and in combination with irradiation (2 and 4 Gy) in nude mice. SK-LMS-1 and SK-UT-1 cells $\left(2 \times 10^{6}\right.$ cells) that had been treated with 5-aza-dC or SGI-110 and irradiated (2 and 4 Gy) were subcutaneously injected into nude mice ( $\mathrm{n}=3$ for each sample group), and the average tumor size was measured two or three times weekly for 7 weeks. Tumor volume and body weight were measured every three days. At the termination of the experiments, all of the mice were sacrificed. (A) Tumor volume of SK-LMS-1 cells (left) and SK-UT-1 cells (right). (B) Representative images of tumor tissues (left) and tumor weight (right). Data are expressed as the mean \pm standard deviation of the three independent experiments. No significant (NS) was not indicated but statistically significant $p$ value was indicated in the graph. ${ }^{*} p<0.05,{ }^{* *} p<0.01,{ }^{* * *} p<0.001$ compared with control or single treatments. 


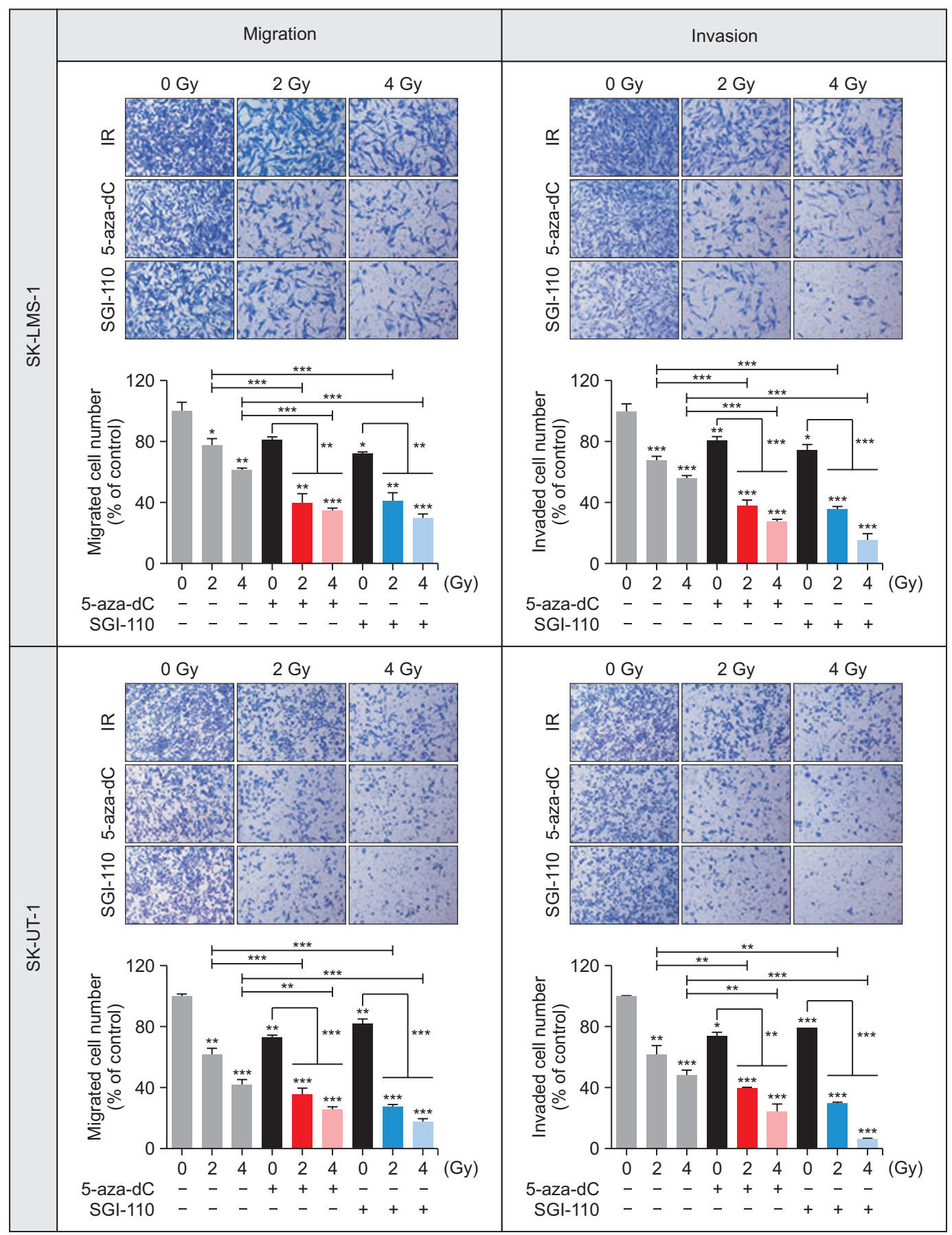

Fig. 4. Combination treatment of 5 -aza-dC or SGI-110 with IR contributes to suppressing invasive traits. Migration and invasion assays in (A) SK-LMS-1 and (B) SK-UT-1 cells. Data are means \pm SE from 3 independent experiments. ${ }^{*} p<0.05,{ }^{* *} p<0.01$, ${ }^{* * *} p<0.001$ compared with control or single treatments.

SGI-110 treatment (Fig. 3). Therefore, these results strongly suggested that the combination treatment of 5-aza-dC or SGI110 with IR affects synergistic tumor growth inhibition both in vitro and in vivo.

To further determine the combined effect of DNA hypomethylating drugs and IR on the metastasis of sarcoma cell lines, the migratory and invasive potential of SK-LMS-1 and
SK-UT-1 cells were assessed using migration and invasion assays. As shown in Fig. 4, the migrating and invading cells in the combination of DNA hypomethylating drugs and IR group were significantly decreased compared with those in the control, 5-aza-dC, SGI-110, or IR alone in both sarcoma cell lines. Our data indicated that the combination of DNA hypomethylating drugs and IR affects sarcoma cell migration and invasion. 
A

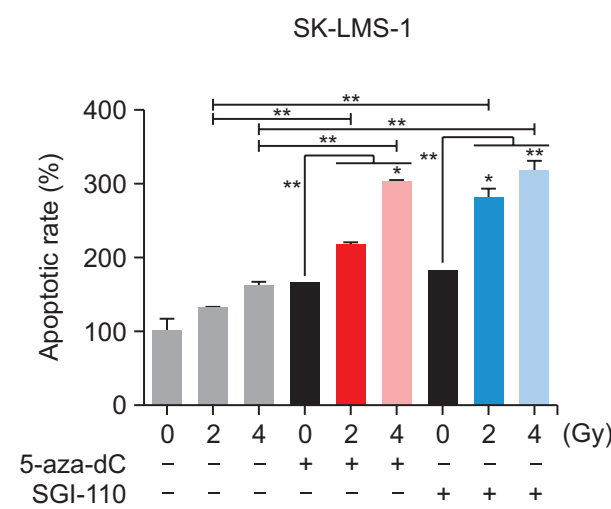

B

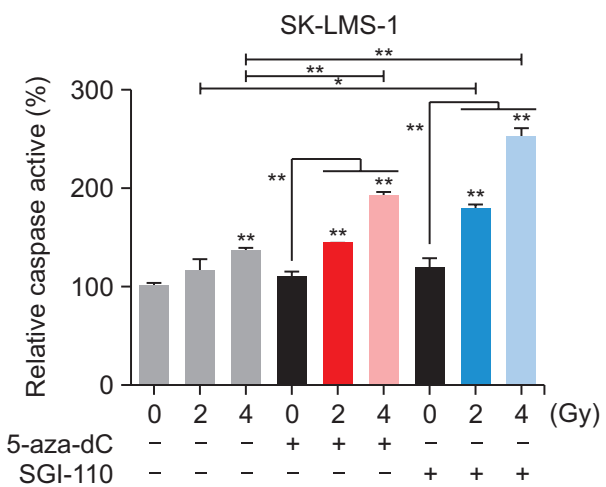

C

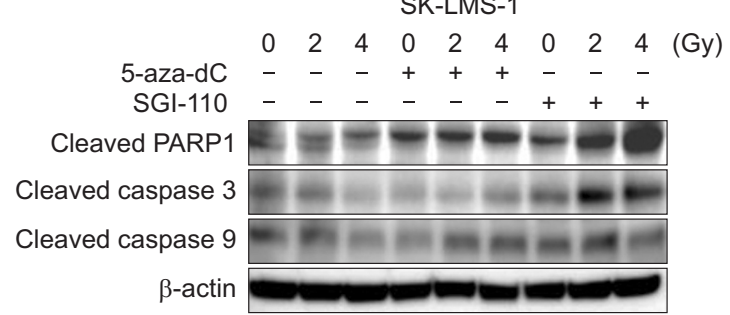

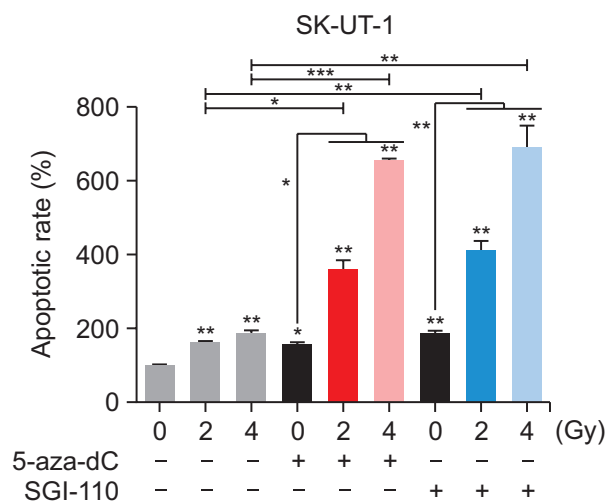

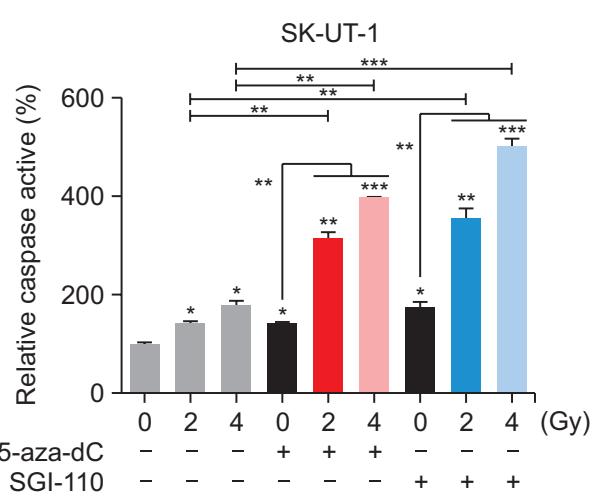

SK-UT-1

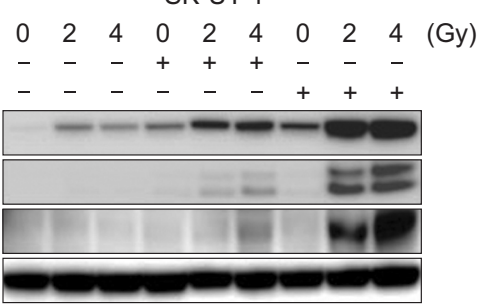

Fig. 5. The combination of 5-aza-dC or SGI-110 and IR contributes to inducing apoptosis in sarcoma cell lines. (A) The levels of apoptosis were measured by using Annexin $\mathrm{V}$ and 7-amino-actinomycin, after which they were analyzed by using a FACS flow cytometer. The levels of apoptosis in SK-LMS-1 and SK-UT-1 cells that were treated with 5-aza-dC $(0.1 \mu \mathrm{M})$ or SGI-110 $(0.1 \mu \mathrm{M})$ either alone or with irradiation $(2$ and $4 \mathrm{~Gy}$ ) are expressed as percentages of the total cell population at both the early and late stages of apoptosis. (B) The activities of caspases 3 and 7 were determined by using the Caspase-Glo assay and are represented as percentages of SK-LMS-1 and SK-UT-1 cells that were treated with 5-aza-dC or SGI-110 either alone or with irradiation (2 and $4 \mathrm{~Gy}$ ), compared with untreated cells. The graph represents data (mean \pm SD) from three independent experiments. No significant (NS) was not indicated but statistically significant $p$ value was indicated in the graph. ${ }^{*} p<0.05,{ }^{* *} p<0.01,{ }^{* * *} p<0.001$. (C) Western blot analysis of the expression levels of apoptosis-associated proteins (cleaved caspase 3, cleaved caspase 9, and cleaved PARP1) in sarcoma cell lines following treatment with 5-aza-dC, SGI-110, or IR alone and in combination.

\section{The combination of DNA hypomethylating drugs and IR contributes to inducing apoptosis in sarcoma cell lines}

To determine the mechanisms that are associated with DNA hypomethylating drug (5-aza-dC and SGI-110)-induced radiosensitivity of sarcoma cells, as well as the inhibition of cell growth both in vitro and in vivo, we employed three different methods to determine the level of cell death due to apoptosis after treatment with either the single agents or the combination treatments. Specifically, we used Annexin V/FITC staining and caspase 3/7 assays (which are known as being key effectors of apoptosis in mammalian cells), in order to determine whether this effect was mediated by caspases; additionally, we used immunoblot analysis to measure apoptotic factors
(Fig. 5).

Annexin $\mathrm{V}$ analysis (Fig. 5) showed a significant increase in apoptotic cells ( 3.9 fold), as well as an increase in caspase 3/7 activities (3.1-fold), in SK-UT-1 cells after treatment with either DNA hypomethylating drugs, IR (2 and $4 \mathrm{~Gy}$ ), or their combination, compared with control cells $(p<0.01)$. In comparison, there was a significant increase in apoptotic cells (1.9-fold) and caspase 3/7 activities (2-fold) in SK-LMS-1 cells after treatment with either DNA hypomethylating drugs, IR (2 and $4 \mathrm{~Gy}$ ), or their combination, compared with control cells $(p<0.05)$. Studies from SK-LMS-1 cells (Fig. 5A) showed that combination treatment resulted in greater cell death than either the uses of DNA hypomethylating drugs or IR alone; the 
fact that apoptosis was not significantly increased suggests that apoptosis is not involved in inducing cell death.

Interestingly, our Western blot analysis demonstrated that the protein levels of caspases 3 and 9 were also higher in cells treated with the combination of 5 -aza-dC and IR than in cells treated with either 5 -aza-dC or IR alone, as well as in control cells. Cleaved PARP1, a well known molecule inducing apoptosis (Kondo and Kondo, 2006; Faustino-Rocha et al., 2013), was also increased in cells treated with 5-aza-dC or IR alone, compared with control cells (Fig. 5C, Supplementary Fig. 2). Strikingly, the combination of SGI-110 treatment with IR led to a similar result, in that the protein levels of the key factors of apoptosis were significantly increased in human sarcoma cells. Taken together, as shown in Fig. 5, based on the synergistic cytotoxicity that was observed with the combined DNA hypomethylating drug treatment and IR exposure in SK-LMS-1 cells (compared to SK-UT-1 cells), it was clear that cell death was present, but not through the mechanism of significant apoptosis. These findings suggest that other mechanisms, such as autophagy, may be associated with promoting cell death.

\section{The combination of DNA hypomethylating drugs and IR contributes to inducing autophagy in sarcoma cell lines}

To examine whether the combination treatment of DNA hypomethylating drugs and IR induced autophagy in SK-LMS-1 cells, we analyzed the levels of autophagic characteristic markers, including ATG3, ATG5, ATG7, ATG12, AGT16L1, LC3, and Beclin by using Western blotting. As shown in Fig. 6 , the expression levels of most of the ATG3, ATG5, ATG7, ATG12, ATG16L1, and Beclin markers were increased by the combination of both DNA hypomethylating drugs and IR, rather than by either treatment with DNA hypomethylating drugs or IR alone. More interestingly, although similar effects were observed at the LC3 protein level, the combination of SGI-110 and IR induced a significant increase in LC3 protein expression, compared with the combination of 5-aza-dC and IR, thus suggesting that $\mathrm{SGI}-110$ is more associated with inducing the autophagy pathway to cancer cell death. LC3-II expression levels were significantly increased in the combination treat- ment of SGI-110 and IR, compared to the control or single agent treatments with either DNA hypomethylating drugs or IR alone (Fig. 6). It has been known that LC3-I converted into LC3-II via lipidation by an ubiquitin-like system during the autophagy process. Afterward, LC3-II stays with autophagosomes until the fusion with lysosomes is completed; therefore, this phenomenon can be used as an autophagy marker (Kondo and Kondo, 2006). Consequently, these results suggested that the combination treatment of DNA hypomethylating drugs and IR in SK-LMS-1 cells can also contribute to autophagic death.

\section{DISCUSSION}

$\mathrm{IR}$ is a well-known genotoxic agent that cause to key molecular damage through both direct and indirect biological mechanisms. Exposure to IR leads to both activation or inactivation of multiple signaling pathways that play key roles in cell survival or death (Goodhead, 1994). In the clinical setting, radiotherapy is most frequently used as the primary or adjuvant therapy in combination with surgery, chemotherapy, or both treatments and widely used as a standard treatment for many types of cancer. Most recently, there has been growing evidence that epigenetic mechanisms, such as DNA methylation and histone modification, are associated with transcriptional gene silencing and may be involved in control of radiosensitivity in cancer cells (De Schutter et al., 2009; Flotho et al., 2009). However, little information is available regarding the influences of DNA hypomethylating drugs on radiosensitization (Dote et al., 2005; Hofstetter et al., 2010). In this context, the combined uses of epigenetic drugs with radiotherapy are particularly interesting and have revealed to improve efficacy in several solid tumors (Dote et al., 2005; De Schutter et al., 2009; Qu et al., 2009; Wang et al., 2012).

Regarding the reversibility of epigenetic modification changes occurring in cancer, it was hypothesized that DNA hypermethylation at promoter $\mathrm{CpG}$ islands was reversed to re-express silenced genes and to reprogram cancer cells to a more normal-like state. Decitabine (5-aza-dC) is a well-known

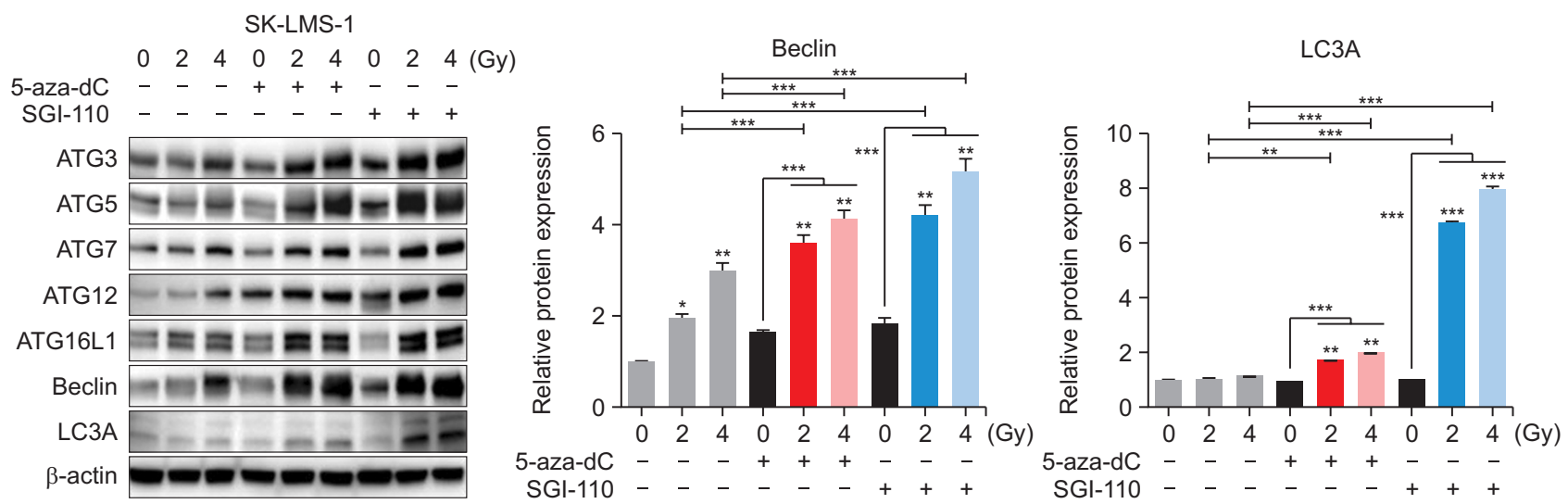

Fig. 6. The combination of 5-aza-dC or SGI-110 and IR induced autophagic death in the SK-LMS-1 cell line. The combination of 5-aza-dC or SGI-110 and IR contributed to inducing autophagy. ATG3, ATG5, ATG7, ATG12, ATG16L1, Beclin, and LC3 expression levels in SKLMS-1 cells treated with 5-aza-dC, SGI-110, or IR alone and in combination was determined by using Western blotting. Representative images of three individual experiments are shown. The statistical data analysis are presented as the means $\pm \mathrm{SD}, \mathrm{n}=3$. ${ }^{*} p<0.05,{ }^{* *} p<0.01$, ${ }^{* * *} p<0.001$ compared with control and single treatments. 
DNA hypomethylating drug that has been successful in the clinic for the treatment of various cancers, such as myelodysplastic syndrome (MDS) (Taby and Issa, 2010) or other leukemia (Issa et al., 2005). Nevertheless, the treatment of solid tumors with a DNA hypomethylating drug (5-aza-dC) as a single agent remains a challenge (van Groeningen et al., 1986). However, there has been evidence that DNA hypomethylating drugs can be effective in inhibiting the solid tumor growth in vitro (Bender et al., 1998; Qin et al., 2009; Tsai et al., 2012). To overcome this problem, there are currently ongoing clinical trials aiming to define whether using hypomethylating drugs as a single-therapy or in combination with other agents, including radiation therapy, can be an effective therapy for solid tumors. Overall, these data indicated that the first-generation hypomethylating drug, decitabine (5-aza-dC), is routinely used for myeloid leukemia (MLLs) and other leukemia. Very recently, SGI-110 (guadecitabine) has been identified as being the most advanced novel and next-generation DNA hypomethylating drug. Guadecitabine was develop to overcome one of the main limits of decitabine and azacitidine, with a key characteristic that it is pharmaceutically stable in the human body because of degradation by cytosine deaminase (CDA). In addition, it is a compound with the structure of decitabine linked to a deoxyguanosine, which made it a more stable compound that is resistant to this degradation (Griffiths et al., 2013). In several studies, guadecitabine demonstrated improved bioavailability and increased half-life acing as a hypomethylating drug (Griffiths et al., 2013).

In the present study, a low dose of 5-aza-dC or SGI-110 $(0.1 \mu \mathrm{M})$ was used in combination with irradiation, although numerous reports have indicated that 5 -aza-dC $(5 \mu \mathrm{M})$ was required to reactivate the expression of most silenced genes (Schuebel et al., 2007). As shown in Fig. 1, we tested the DNMT1 expression level with different doses $(0.1,0.5$, and $1 \mu \mathrm{M})$ of 5 -aza-dC or SGI-110 in sarcoma cell lines and observed that a lower dose $(0.1 \mu \mathrm{M})$ of DNA hypomethylating drugs was sufficient to inhibit global DNMT1 levels in sarcoma cell lines. In addition, we found that a lower dose of 5-aza$\mathrm{dC}$ or SGI-110 $(0.1 \mu \mathrm{M})$ and a nontoxic level of IR (2 Gy or 4 Gy) were sufficient to induce anticancer effects in sarcoma cell lines. In this regard, our data strongly support the hypothesis that giving lower doses of the drug would lead to more effective for therapeutic approach and improved clinical trials (Issa and Kantarjian, 2009).

Here, we used two different DNA hypomethylating drugs. Specifically, 5-aza-dC (decitabine) is a well-known demethylating drug and FDA approved agent for MDS, and the other drug is a next generation DNA hypomethylating drug that has been recently developed and is currently being investigated in clinical trials. Unlike previous studies, although we used low doses $(0.1 \mu \mathrm{M})$ of both DNA hypomethylating drugs in sarcoma cell lines, the global level of DNMT1 protein was significantly decreased (Fig. 1). The data presented in this study indicates that 5-aza-dC and SGI-110 increased radiosensitivity in two sarcoma cell lines (SK-LMS-1 and SK-UT-1), which is consistent with reports on zeburaline, another DNA hypomethylating drug (Dote et al., 2005). We also found that both DNA inhibitors increased radiation-induced cell death, as has been evaluated in in vitro and in vivo xenograft models. In addition, it was interesting to observe that the combination treatment of DNA hypomethylating drugs and IR enhanced the cell death that is associated with both apoptosis and autophagy in sar- coma cell lines.

Autophagy has been classified as a second form of programmed cell death. First, it was observed in cells that were exposed to starvation, which implicating that it presumably a cell protective mechanism to survive during starvation or when they were exposed to environmental toxicity. Therefore, it thought to be that autophagy enhances both cancer cell death and survival (Levine, 2007; Dalby et al., 2010). Kroemer and Levine (2008) suggest two possible explanations of the biological role for autophagy in cell death. One is that autophagy only coexists with cell death, and the other is that autophagy could be a cell death mechanism (Kroemer and Levine, 2008). Collectively, the cellular function of autophagy in human cancer still remains controversial.

Little is known about the cellular mechanisms of the radiosensitivity that is induced by epigenetic inhibitors. Recently, we and other researchers have reported that the combinations of epigenetic inhibitors with radiotherapy are capable of increasing apoptosis in several solid tumors and is regarded as a potential mechanism for radiosensitization (Qiu et al., 2009; Kim et al., 2012, 2014; Kwon et al., 2017). Autophagy has been suggested as a novel mechanism of eliciting synergistic cytotoxicity via several chemotherapeutic agents in sarcomas in vitro or in vivo (Lambert et al., 2008; Li et al., 2013). Here we present evidence that the combination treatment of DNA hypomethylating drugs and radiation is also capable of increasing autophagic cell death in sarcoma cell lines.

In the beginning of this study, we started analyzing the radiation response in four different sarcomas that originated from different tissues, such as bone, uterine, and vulva tissues; however, we ended up studying the use of two different cell lines, including uterine leiomyosarcomas (ULMSs). ULMS is a rare gynecologic malignancy with a low survival rate. Although it is a rare tumor that accounts for less than $1 \%$ of all uterine malignancies, over two thirds of patients with ULMS that has extended among the uterus experience tumor recurrence after initial chemotherapy (Dinh et al., 2004). Human ULMS occurs infrequently and exhibit obstacles for understanding the etiology of the disease making it difficult to develop efficient therapies. Therefore, our experimental findings suggest that the combined effect of DNA hypomethylating drugs and radiation might provide a new approach for the development of a new therapeutic strategy.

\section{CONFLICT OF INTEREST}

The author declares no conflict of interest.

\section{ACKNOWLEDGMENTS}

This work was supported by grant from the National Research Foundation of Korea (NRF) funded by the Korean government (MSIT) (NRF-2020M2C8A2069356).

\section{REFERENCES}

Bender, C. M., Pao, M. M. and Jones, P. A. (1998) Inhibition of DNA methylation by 5-aza-2'-deoxycytidine suppresses the growth of human tumor cell lines. Cancer Res. 58, 95-101. 
Brennan, M. F., Antonescu, C. R., Moraco, N. and Singer, S. (2014) Lessons learned from the study of 10,000 patients with soft tissue sarcoma. Ann. Surg. 260, 416-422.

Dalby, K., Tekedereli, I., Lopez-Berestein, G. and Ozpolat, B. (2010) Targeting the pro-death and pro-survival functions of autophagy as novel therapeutic strategies in cancer. Autophagy 6, 322-329.

De Schutter, H., Kimpe, M., Isebaert, S. and Nuyts, S. (2009) A systematic assessment of radiation dose enhancement by 5-Aza-2'deoxycytidine and histone deacetylase inhibitors in head-and-neck squamous cell carcinoma. Int. J. Radiat. Oncol. Biol. Phys. 73, 904-912.

Dinh, T. A., Oliva, E. A., Fuller, A. F., Jr., Lee, H. and Goodman, A. (2004) The treatment of uterine leiomyosarcoma. Results from a 10-year experience (1990-1999) at the Massachusetts General Hospital. Gynecol. Oncol. 92, 648-652.

Dote, H., Cerna, D., Burgan, W. E., Carter, D. J., Cerra, M. A., Hollingshead, M. G., Camphausen, K. and Tofilon, P. J. (2005) Enhancement of in vitro and in vivo tumor cell radiosensitivity by the DNA methylation inhibitor zebularine. Clin. Cancer Res. 11, 4571-4579.

Faustino-Rocha, A., Oliveira, P. A., Pinho-Oliveira, J., TeixeiraGuedes, C., Soares-Maia, R., da Costa, R. G., Colaco, B., Pires, M. J., Colaco, J., Ferreira, R. and Ginja, M. (2013) Estimation of rat mammary tumor volume using caliper and ultrasonography measurements. Lab. Anim. (N.Y.) 42, 217-224.

Flotho, C., Claus, R., Batz, C., Schneider, M., Sandrock, I., Ihde, S., Plass, C., Niemeyer, C. M. and Lübbert, M. (2009) The DNA methyltransferase inhibitors azacitidine, decitabine and zebularine exert differential effects on cancer gene expression in acute myeloid leukemia cells. Leukemia 23, 1019-1028.

Gage, M. M., Nagarajan, N., Ruck, J. M., Canner, J. K., Khan, S., Giuliano, K., Gani, F., Wolfgang, C., Johnston, F. M. and Ahuja, N. (2019) Sarcomas in the United States: recent trends and a call for improved staging. Oncotarget 10, 2462-2474.

Goodhead, D. T. (1994) Initial events in the cellular effects of ionizing radiations: clustered damage in DNA. Int. J. Radiat. Biol. 65, 7-17.

Griffiths, E. A., Choy, G., Redkar, S., Taverna, P., Azab, M. and Karpf, A. R. (2013) SGI-110: DNA methyltransferase inhibitor oncolytic. Drugs Future 38, 535-543.

Hofstetter, B., Niemierko, A., Forrer, C., Benhattar, J., Albertini, V., Pruschy, M., Bosman, F. T., Catapano, C. V. and Ciernik, I. F. (2010) Impact of genomic methylation on radiation sensitivity of colorectal carcinoma. Int. J. Radiat. Oncol. Biol. Phys. 76, 1512-1519.

Issa, J. P. J. and Kantarjian, H. M. (2009) Targeting DNA methylation. Clin. Cancer Res. 15, 3938-3946.

Issa, J. P., Gharibyan, V., Cortes, J., Jelinek, J., Morris, G., Verstovsek, S., Talpaz, M., Garcia-Manero, G. and Kantarjian, H. M. (2005) Phase II study of low-dose decitabine in patients with chronic myelogenous leukemia resistant to imatinib mesylate. J. Clin. Oncol. 23, 3948-3956.

Jain, S., Xu, R., Prieto, V. G. and Lee, P. (2010) Molecular classification of soft tissue sarcomas and its clinical applications. Int. J. Clin. Exp. Pathol. 3, 416-428.

Kaminskas, E., Farrell, A., Abraham, S., Baird, A., Hsieh, L. S., Lee, S L., Leighton, J. K., Patel, H., Rahman, A., Sridhara, R., Wang, Y. C. and Pazdur, R. (2005) Approval summary: azacitidine for treatment of myelodysplastic syndrome subtypes. Clin. Cancer Res. 11, 3604-3608.

Kelly, T. K., De Carvalho, D. D. and Jones, P. A. (2010) Epigenetic modifications as therapeutic targets. Nat. Biotech. 28, 1069-1078.

Kim, H., Kim, J., Chie, E., Park, D., Kim, I. and Kim, I. (2012) DNMT (DNA methyltransferase) inhibitors radiosensitize human cancer cells by suppressing DNA repair activity. Radiat. Oncol. 7, 39.

Kim, J. G., Bae, J. H., Kim, J. A., Heo, K., Yang, K. and Yi, J. M. (2014) Combination effect of epigenetic regulation and ionizing radiation in colorectal cancer cells. PLoS ONE 9, e105405.

Kneisl, J. S., Coleman, M. M. and Raut, C. P. (2014) Outcomes in the management of adult soft tissue sarcomas. J. Surg. Oncol. 110, 527-538.

Kondo, Y. and Kondo, S. (2006) Autophagy and cancer therapy. Au- tophagy 2, 85-90.

Kristensen, L. S., Nielsen, H. M. and Hansen, L. L. (2009) Epigenetics and cancer treatment. Eur. J. Pharmacol. 625, 131-142.

Kroemer, G. and Levine, B. (2008) Autophagic cell death: the story of a misnomer. Nat. Rev. Mol. Cell Biol. 9, 1004-1010.

Kwon, H. M., Kang, E. J., Kang, K., Kim, S. D., Yang, K. and Yi, J. M. (2017) Combinatorial effects of an epigenetic inhibitor and ionizing radiation contribute to targeted elimination of pancreatic cancer stem cell. Oncotarget 8, 89005-89020.

Lambert, L. A., Qiao, N., Hunt, K. K., Lambert, D. H., Mills, G. B., Meijer, L. and Keyomarsi, K. (2008) Autophagy: a novel mechanism of synergistic cytotoxicity between doxorubicin and roscovitine in a sarcoma model. Cancer Res. 68, 7966-7974.

Levine, B. (2007) Autophagy and cancer. Nature 446, 745-747.

Li, B., Takeda, T., Tsuiji, K., Wong, T. F., Tadakawa, M., Kondo, A., Nagase, S. and Yaegashi, N. (2013) Curcumin induces cross-regulation between autophagy and apoptosis in uterine leiomyosarcoma cells. Int. J. Gynecol. Cancer 23, 803-808.

Qin, T., Jelinek, J., Si, J., Shu, J. and Issa, J. P. J. (2009) Mechanisms of resistance to 5 -aza-2'-deoxycytidine in human cancer cell lines. Blood 113, 659-667.

Qiu, H., Yashiro, M., Shinto, O., Matsuzaki, T. and Hirakawa, K. (2009) DNA methyltransferase inhibitor 5-aza-CdR enhances the radiosensitivity of gastric cancer cells. Cancer Sci. 100, 181-188.

Sato, T., Issa, J. P. J. and Kropf, P. (2017) DNA hypomethylating drugs in cancer therapy. Cold Spring Harb. Perspect. Med. 7, a026948.

Schaefer, I. M., Cote, G. M. and Hornick, J. L. (2017) Contemporary sarcoma diagnosis, genetics, and genomics. J. Clin. Oncol. 36, $101-110$.

Schuebel, K. E., Chen, W., Cope, L., Glöckner, S. C., Suzuki, H., Yi, J. M., Chan, T. A., Neste, L. V., Criekinge, W. V., Bosch, S. V., van Engeland, M., Ting, A. H., Jair, K., Yu, W., Toyota, M., Imai, K., Ahuja, N., Herman, J. G. and Baylin, S. B. (2007) Comparing the DNA hypermethylome with gene mutations in human colorectal cancer. PLoS Genet. 3, 1709-1723.

Ta, H. T., Dass, C. R., Choong, P. F. M. and Dunstan, D. E. (2009) Osteosarcoma treatment: state of the art. Cancer Metastasis Rev. 28, 247-263.

Taby, R. and Issa, J. P. J. (2010) Cancer epigenetics. CA Cancer J. Clin. 60, 376-392.

Toro, J. R., Travis, L. B., Wu, H. J., Zhu, K., Fletcher, C. D. M. and Devesa, S. S. (2006) Incidence patterns of soft tissue sarcomas, regardless of primary site, in the surveillance, epidemiology and end results program, 1978-2001: an analysis of 26,758 cases. Int. J. Cancer 119, 2922-2930.

Trans-Atlantic Retroperitoneal Sarcoma Working Group (2018) Management of metastatic retroperitoneal sarcoma: a consensus approach from the Trans-Atlantic Retroperitoneal Sarcoma Working Group (TARPSWG). Ann. Oncol. 29, 857-871.

Tsai, H. C., Li, H., Van Neste, L., Cai, Y., Robert, C., Rassool, F. V., Shin, J. J., Harbom, K. M., Beaty, R., Pappou, E., Harris, J., Yen, R. W., Ahuja, N., Brock, M. V., Stearns, V., Feller-Kopman, D., Yarmus, L. B., Lin, Y. C., Welm, A. L., Issa, J. P., Minn, I., Matsui, W., Jang, Y. Y., Sharkis, S. J., Baylin, S. B. and Zahnow, C. A. (2012) Transient low doses of DNA-demethylating agents exert durable antitumor effects on hematological and epithelial tumor cells. Cancer Cell 21, 430-446.

van Groeningen, C. J., Leyva, A., Brien, A. M. P., Gall, H. E. and Pinedo, H. M. (1986) Phase I and pharmacokinetic study of 5-aza-2'deoxycytidine (NSC 127716) in cancer patients. Cancer Res. 46, 4831-4836.

Wang, L., Zhang, Y., Li, R., Chen, Y., Pan, X., Li, G., Dai, F. and Yang, J. (2012) 5-Aza-2'-deoxycytidine enhances the radiosensitivity of breast cancer cells. Cancer Biother. Radiopharm. 28, 34-44.

Yoo, C. B. (2007) Delivery of 5-aza-2[prime]-deoxycytidine to cells using oligodeoxynucleotides. Cancer Res. 67, 6400-6408.

Yoo, C. B. and Jones, P. A. (2006) Epigenetic therapy of cancer: past, present and future. Nat. Rev. Drug Discov. 5, 37-50. 